'Departamento de Ortopedia, Facultad de Medicina, Pontificia Universidad Católica de Chile. Santiago, Chile.

2Departamento de Matemáticas, Universidad Técnica Federico Santa María. Santiago, Chile. aMatrona. ${ }^{b}$ Enfermera. 'Estadística.

Trabajo no recibió financiamiento. Los autores declaran no tener conflictos de interés.

Recibido el 23 de marzo de 2018 , aceptado el 14 de noviembre de 2018.

Correspondencia a: Dr. Sebastián Irarrázaval Pontificia Universidad Católica de Chile, Facultad de Medicina, Departamento de Ortopedia y Traumatología. Diagonal Paraguay 362, Santiago. Fono: 56-2-3543467 Código Postal 8330077. sirarraz@med.puc.cl

\section{¿Cómo es la calidad de vida reportada por los estudiantes de Medicina?}

\author{
LUIS IRRIBARRA T. ${ }^{1}$, PAMELA MERY I. ${ }^{1, a}$, \\ MARÍA JESÚS LIRA S. ${ }^{1, b}$, MAURICIO CAMPOS D. ${ }^{1}$, \\ FRANCISCA GONZÁLEZ L. ${ }^{2, c}$, SEBASTIÁN IRARRÁZAVAL D. ${ }^{1}$

\section{Quality of life scores among 411 medical students}

Background: The high academic burden may hamper the quality of life of medical students. Aim: To evaluate the quality of life (QOL) for medical students attending a Chilean university. Material and Methods: Four hundred eleven medical students aged $22 \pm 2$ years (51\% women), studying in Santiago, Chile, answered online a validated Spanish version of the WHOQOL-BREF quality of life survey (scored from 0 to 100). Overall scores were assessed for the questionnaire domains Physical health, Psychological health, Interpersonal relationships, and Environment. Results: The global scores were 65.1 for Physical health, 63.1 for Psychological health, 61.3 for Interpersonal relationships and 67.2 for Environment. Students in clinical practice, females, those with sedentary behaviors and consuming modafinil had lower Physical health scores. Students coming from outside Santiago, with sedentary behaviors and who consumed modafinil had poorer Psychological health scores. Students coming from outside Santiago, males and those with sedentary behaviors had Lower Interpersonal relationship scores. Environment scores were also lower among students who were sedentary or from outside Santiago. Conclusions: The variables that had a greater negative impact in the quality of life of these students were the transition from theoretical courses to clinical practice, being from outside Santiago, being overweight or obese and consuming modafinil. Students that were physically active had better quality of life scores.

(Rev Med Chile 2018; 146: 1294-1303)

Key words: Education, Medical; Quality of Life; Students, Medical.
L a calidad de vida $(\mathrm{CV})$ es un tema relevante en la literatura médica ${ }^{1-5}$. La Organización Mundial de la Salud (OMS) la define como "la percepción del individuo de su posición en la vida, en el contexto cultural y el sistema de valores en que vive, en relación con sus metas, objetivos, expectativas, valores y preocupaciones". En el caso de los estudiantes de Medicina, puede verse afectada por la alta exigencia académica y emocional en cada etapa de formación, escaso tiempo libre, contacto con enfermedades y muerte ${ }^{1-3}$.

Estudios realizados en países como China,
Brasil y Nueva Zelanda señalan que los estudiantes de Medicina estarían más propensos a presentar ansiedad, depresión, burnout y estrés, en comparación a otros estudiantes universitarios. Esto tiene una alta relevancia, ya que una peor calidad de vida no sólo afecta el rendimiento académico del estudiante, sino que además podría influir en el cuidado de los pacientes ${ }^{7,8}$.

Datos de la literatura chilena en relación a evaluación de estudiantes del área de la salud muestran una prevalencia de $36 \%$ de síntomas de estrés. En estudios específicos realizados a es- 
tudiantes de medicina muestran una prevalencia de depresión y ansiedad cercana a $40 \%$ y $13 \%$ de burnout severo ${ }^{9-12}$.

Hasta la fecha no se han publicado estudios que describan la CV en estudiantes de Medicina chilenos, por lo que se desconoce la realidad local de este problema. El objetivo de este estudio es evaluar la calidad de vida de los estudiantes de Medicina de una universidad chilena, y sus factores asociados, considerando el ciclo completo de formación (de primer a séptimo año).

\section{Material y Método}

\section{Estudio transversal}

Se invitó a participar a todos los estudiantes de Medicina en la Pontificia Universidad Católica de Chile durante el año 2014. El criterio de inclusión fue que los estudiantes estuvieran matriculados y cursando la carrera de Medicina.

Se envió una encuesta online vía correo electrónico institucional, con respuesta anónima. Se evaluó, a través de un cuestionario de selección múltiple, los datos socio-demográficos (edad, índice de masa corporal -IMC-, sexo, consumo de drogas, consumo de modafinilo, región geográfica de origen y actividad física). La CV se midió a través del cuestionario WHOQOL-BREF' ${ }^{6}$. Para el análisis de datos se agruparon a los estudiantes en tres etapas de formación según los años de estudio: Ciencias Básicas ( $1^{\circ}$ y $2^{\circ}$ año), Cursos Clínicos ( $3^{\circ}$ a $5^{\circ}$ año) e Internados ( $6^{\circ}$ y $7^{\circ}$ año) (Tabla 1$)$. El proyecto fue aprobado por el Comité de Ética Científico de la Facultad de Medicina de la Pontificia Universidad Católica de Chile ( $\left.\mathrm{N}^{\circ} 14-364\right)$.

\section{Instrumento WHOQOL-BREF}

El centro WHOQOL center autorizó y facilitó el instrumento en español, el cual consiste en 26 preguntas. Las dos primeras evalúan $\mathrm{CV}$ global y satisfacción respecto al estado de salud y las 24 siguientes evalúan cuatro dimensiones: Salud Física, Salud Psicológica, Relaciones Interpersonales y Entorno. Las respuestas son de tipo Likert (escala), con 5 opciones. Posteriormente, hay una conversión de puntaje de 0 a 100, donde puntuaciones mayores indican mejor CV. En las preguntas "dolor", "dependencia a medicamentos" y "sentimientos negativos" se realizó una inversión de la escala numérica para poder ser comparadas.

\section{Análisis estadístico}

Se utilizó la prueba de chi cuadrado para variables categóricas, $t$-student y ANOVA para medias. Se realizó regresión lineal multivariante paso a paso utilizando las mismas variables analizadas de forma independiente, reportando el efecto de cada variable sobre los puntajes en cada dominio de CV. Se calculó alpha de Cronbach's para evaluar la consistencia interna de cada dimensión. Se realizó un análisis factorial confirmatorio para verificar si el modelo teórico de cuatro dimensiones se ajusta a los datos. El análisis estadístico fue realizado con el software SPSS (v22.0), considerando significancia $<0,05$.

\section{Resultados}

\section{Características generales}

De un total de 856 estudiantes de Medicina, 411 (48\%) completaron la encuesta, de los cuales el 50,6\% correspondió al sexo femenino. La edad promedio de la muestra fue $22 \pm 2,39$ años (18-35). Las características generales de los estudiantes están descritas en la Tabla 1. Respecto al IMC, 1,95\% presentó bajo peso $(<18,5), 20,9 \%$ sobrepeso $(25-29,99)$ y $2,9 \%$ obesidad $(\geq 30)$. Los hombres presentaron un OR 2,29 veces mayor (IC 95\% 1,36-3,90) que las mujeres de presentar sobrepeso.

En el consumo de drogas y modafinilo durante el último año, el consumo fue significativamente mayor en los Cursos Clínicos (Tabla 1). No se encontraron diferencias significativas respecto a los diferentes hábitos al analizar por las variables estudiadas.

\section{Análisis de los resultados de la escala de calidad de vida WHOQL-BREF}

Los resultados del cuestionario WHOQL-BREF se analizaron según las variables etapa de formación, actividad física, región geográfica de origen y sexo (Tabla 2).

\section{Calidad de Vida según la variable "etapa de formación"}

En la pregunta ¿cómo puntuaría su calidad de vida? y respecto a la satisfacción de salud, la etapa de formación Ciencias Básicas obtuvo un puntaje significativamente mayor que las otras etapas (Tabla 2). 
a.

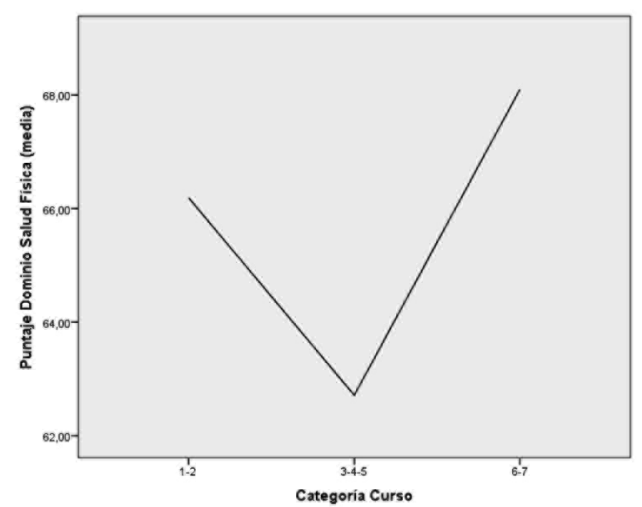

c.

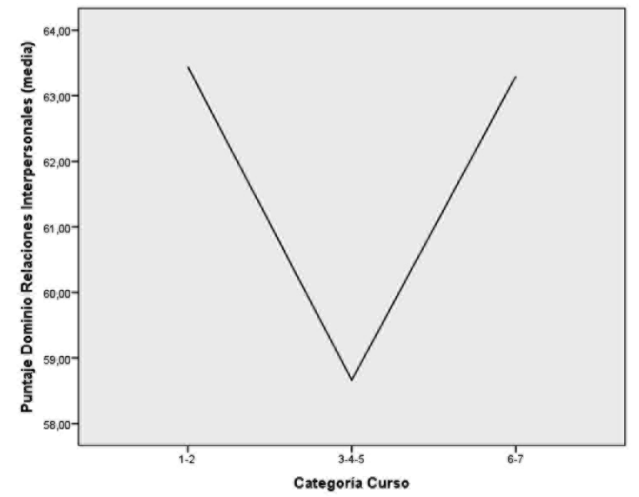

b.

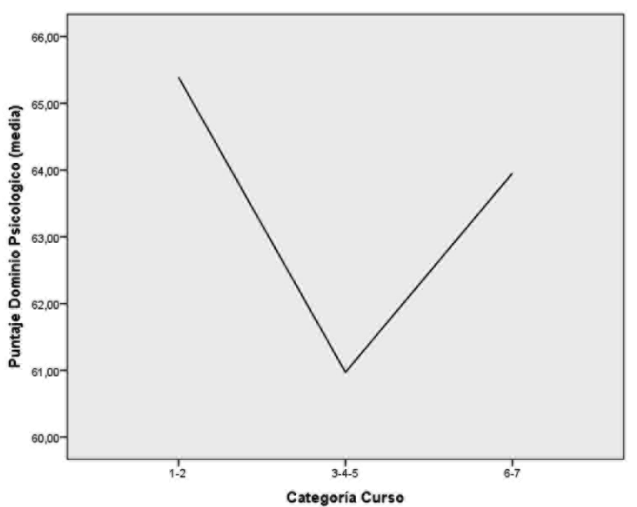

d.

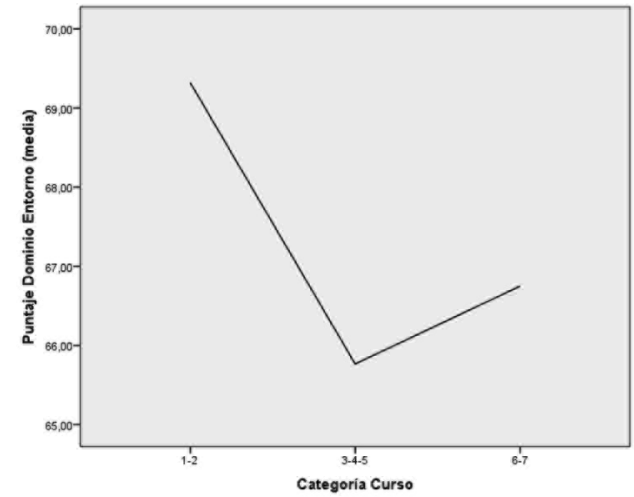

Figura 1. Puntajes WHOQOL-BREF por dominio según categoría curso. a. Dominio Salud Física; b. Dominio Psicológico, c. Dominio Relaciones interpersonales, d. Dominio Entorno. Categorías curso $1-2^{\circ}$ año (cursos ciencias básicas), 3-4-5 año (cursos clínicos), 6-7º año (internados).

En salud física destaca un puntaje significativamente menor en los Cursos Clínicos al compararlos con los Internados, observándose una mayor diferencia en la satisfacción respecto al sueño (33 vs 45 puntos) (Tablas 2 y 3 ).

En salud psicológica, relaciones interpersonales y entorno no se encontró diferencias en el puntaje global (Tablas 2 y 3 ).

2. Calidad de Vida según la variable "región geográfica de origen"

Los estudiantes con origen geográfico fuera de la Región Metropolitana (RM) tuvieron puntajes significativamente más bajos en salud psicológica, relaciones interpersonales y entorno (Tablas 2 y 3 ), sin embargo, no hubo diferencias en los puntajes globales de salud física (Tabla 3).

3. Calidad de Vida según la variable "sexo"

Los hombres presentaron mejores puntajes en salud física en comparación a las mujeres, quienes a su vez, presentaron mejores puntajes en relaciones interpersonales. En salud psicológica no se observó diferencias en los puntajes globales según sexo, sin embargo, las mujeres presentaron peores puntajes en las preguntas específicas a pensamientos negativos e impresión de la propia imagen corporal (Tabla 3). 
Tabla 1. Características generales [n (\%)] de los estudiantes de Medicina evaluados

\begin{tabular}{|c|c|c|c|c|c|}
\hline Variable & $\begin{array}{c}\text { Total } \\
411\end{array}$ & $\begin{array}{l}\text { Ciencias } \\
\text { básicas } \\
139(53,3)\end{array}$ & $\begin{array}{l}\text { Cursos } \\
\text { clínicos } \\
178(48,6)\end{array}$ & $\begin{array}{c}\text { Internados } \\
94(38,7)\end{array}$ & Valor $\mathbf{p}^{*}$ \\
\hline $\begin{array}{l}\text { Sexo } \\
\text { Femenino } \\
\text { Masculino } \\
\text { Valor } p\end{array}$ & $\begin{array}{l}208(50,6) \\
203(40,4)\end{array}$ & $\begin{array}{l}82(59,0) \\
57(41,0)\end{array}$ & $\begin{array}{r}75(42,13) \\
103(57,87)\end{array}$ & $\begin{array}{l}51(54,36) \\
43(45,74)\end{array}$ & $<0,05$ \\
\hline $\begin{array}{l}\text { Región de origen } \\
\text { Región Metropolitana } \\
\text { Otra Región }\end{array}$ & $\begin{array}{l}274(66,67) \\
137(33,33)\end{array}$ & $\begin{array}{l}87(62,59) \\
52(37,41)\end{array}$ & $\begin{array}{r}116(65,17) \\
62(34,83)\end{array}$ & $\begin{array}{l}71(75,53) \\
23(24,47)\end{array}$ & NS \\
\hline $\begin{array}{l}\text { Actividad física } \\
\text { Sí } \\
\text { No }\end{array}$ & $\begin{array}{l}263(64,0) \\
148(36,0)\end{array}$ & $\begin{array}{l}96(69,06) \\
43(30,94)\end{array}$ & $\begin{array}{r}104(58,43) \\
74(41,57)\end{array}$ & $\begin{array}{l}63(67,02) \\
31(32,98)\end{array}$ & NS \\
\hline $\begin{array}{l}\text { Consumo de tabaco } \\
\text { Sí } \\
\text { No }\end{array}$ & $\begin{array}{r}36(8,76) \\
375(91,24)\end{array}$ & $\begin{array}{r}10(7,19) \\
129(92,81)\end{array}$ & $\begin{array}{r}18(10,11) \\
160(89,89)\end{array}$ & $\begin{array}{rr}8 & (8,51) \\
86 & (91,49)\end{array}$ & NS \\
\hline $\begin{array}{l}\text { Consumo de drogas durante el último año } \\
\text { Sí } \\
\text { No }\end{array}$ & $\begin{array}{l}144(35,04) \\
267(64,96)\end{array}$ & $\begin{array}{l}45(32,37) \\
94(67,63)\end{array}$ & $\begin{array}{l}79(44,38) \\
99(55,62)\end{array}$ & $\begin{array}{l}23(24,47) \\
71(75,53)\end{array}$ & $<0,05$ \\
\hline $\begin{array}{l}\text { Consumo de Modafinilo durante el último año } \\
\text { Sí } \\
\text { No }\end{array}$ & $\begin{array}{r}71(17,27) \\
340(82,73)\end{array}$ & $\begin{array}{r}6(4,32) \\
133(95,68)\end{array}$ & $\begin{array}{r}45(25,28) \\
133(74,72)\end{array}$ & $\begin{array}{l}20(21,28) \\
74(78,72)\end{array}$ & $<0,01$ \\
\hline
\end{tabular}

*Test chi cuadrado. NS: valor p no significativo.

Tabla 2. Puntajes WHOQOL-BREF según categoría Curso, Región geográfica de Origen, Sexo, Actividad Física y Consumo de Modafinilo

\begin{tabular}{|c|c|c|c|c|c|c|c|}
\hline Variable & n (\%) & $\begin{array}{c}\text { Puntuación } \\
\text { CV }\end{array}$ & $\begin{array}{l}\text { Satisfacción } \\
\text { de salud }\end{array}$ & Salud física & $\begin{array}{c}\text { Salud } \\
\text { psicológica }\end{array}$ & $\begin{array}{c}\text { Relaciones } \\
\text { inter- } \\
\text { personales }\end{array}$ & Entorno \\
\hline Puntaje global & $411(100)$ & $68,8 \pm 28,8$ & $65,4 \pm 25,3$ & $65,1 \pm 14,7$ & $63,1 \pm 17,9$ & $61,3 \pm 23,3$ & $67,2 \pm 15,8$ \\
\hline $\begin{array}{l}\text { Categoría curso } \\
\text { Ciencias básicas } \\
\text { Cursos clínicos } \\
\text { Internados } \\
\text { Valor p }\end{array}$ & $\begin{array}{r}137(34,0) \\
177(43,9) \\
89(22,0)\end{array}$ & $\begin{array}{c}76,4 \pm 20,5^{a} \\
64,1 \pm 23,4 \\
66,3 \pm 21,9 \\
<0,001\end{array}$ & $\begin{array}{c}70,3 \pm 24,4^{b} \\
60,9 \pm 26,6^{b} \\
66,6 \pm 22,4 \\
0,004\end{array}$ & $\begin{array}{c}66,2 \pm 14,0 \\
62,7 \pm 14,9^{b} \\
68,1 \pm 14,5^{b} \\
0,01\end{array}$ & $\begin{array}{c}65,4 \pm 16,1 \\
60,9 \pm 19,4 \\
63,9 \pm 17,1 \\
0,084\end{array}$ & $\begin{array}{c}63,4 \pm 22,1 \\
58,7 \pm 24,1 \\
63,3 \pm 23,2 \\
0,131\end{array}$ & $\begin{array}{c}69,3 \pm 15,3 \\
65,8 \pm 16,5 \\
66,7 \pm 14,8 \\
0,136\end{array}$ \\
\hline $\begin{array}{l}\text { Región origen } \\
\text { Metropolitana } \\
\text { Otras } \\
\text { Valor } p\end{array}$ & $\begin{array}{l}268(66,7) \\
134(33,3)\end{array}$ & $\begin{array}{c}69,7 \pm 23,1 \\
67,1 \pm 22,3 \\
0,274\end{array}$ & $\begin{array}{c}66,9 \pm 24,9 \\
62,1 \pm 25,8 \\
0,070\end{array}$ & $\begin{array}{c}65,8 \pm 14,3 \\
63,5 \pm 15,3 \\
0,143\end{array}$ & $\begin{array}{c}64,6 \pm 17,1^{b} \\
59,9 \pm 19,0^{b} \\
0,013\end{array}$ & $\begin{array}{c}63,1 \pm 22,9^{b} \\
57,7 \pm 23,7^{b} \\
0,029\end{array}$ & $\begin{array}{c}68,5 \pm 16,0^{b} \\
64,4 \pm 14,9^{b} \\
0,015\end{array}$ \\
\hline $\begin{array}{l}\text { Sexo } \\
\text { Mujer } \\
\text { Hombre } \\
\text { Valor p }\end{array}$ & $\begin{array}{l}206(51,1) \\
197(48,9)\end{array}$ & $\begin{array}{c}70,0 \pm 21,9 \\
67,6 \pm 23,6 \\
0,284\end{array}$ & $\begin{array}{c}65,7 \pm 26,1 \\
65,1 \pm 24,5 \\
0,819\end{array}$ & $\begin{array}{c}63,2 \pm 14,7^{b} \\
67,0 \pm 14,3^{b} \\
0,009\end{array}$ & $\begin{array}{c}62,8 \pm 16,7 \\
63,4 \pm 19,1 \\
0,708\end{array}$ & $\begin{array}{c}64,3 \pm 22,5^{b} \\
58,1 \pm 23,8^{b} \\
0,008\end{array}$ & $\begin{array}{c}67,6 \pm 16,2 \\
66,7 \pm 15,3 \\
0,572\end{array}$ \\
\hline $\begin{array}{l}\text { Actividad física } \\
\text { Sí } \\
\text { No } \\
\text { Valor p }\end{array}$ & $\begin{array}{l}258(64,0) \\
145(36,0)\end{array}$ & $\begin{array}{c}72,6 \pm 21,4^{b} \\
62,2 \pm 23,7^{b} \\
\quad<0,001\end{array}$ & $\begin{array}{c}69,8 \pm 23,9^{b} \\
57,6 \pm 25,9^{b} \\
\quad<0,001\end{array}$ & $\begin{array}{c}67,6 \pm 14,5^{b} \\
60,5 \pm 13,9^{b} \\
\quad<0,001\end{array}$ & $\begin{array}{l}66,6 \pm 16,5^{b} \\
56,9 \pm 18,5^{b} \\
\quad<0,001\end{array}$ & $\begin{array}{c}64,1 \pm 22,2^{b} \\
56,2 \pm 24,3^{b} \\
0,01\end{array}$ & $\begin{array}{c}69,1 \pm 15,0^{b} \\
63,6 \pm 16,4^{b} \\
0,01\end{array}$ \\
\hline $\begin{array}{l}\text { Modafinilo } \\
\text { Sí } \\
\text { No } \\
\text { Valor p }\end{array}$ & $\begin{array}{r}70(17,1) \\
333(81,2)\end{array}$ & $\begin{array}{c}64,4 \pm 23,8 \\
69,8 \pm 22,5 \\
0,072\end{array}$ & $\begin{array}{c}57,4 \pm 23,3^{b} \\
67,1 \pm 25,4^{b} \\
0,003\end{array}$ & $\begin{array}{c}59,7 \pm 16,3^{b} \\
66,2 \pm 14,1^{b} \\
0,001\end{array}$ & $\begin{array}{c}57,3 \pm 19,6^{b} \\
64,4 \pm 17,3^{b} \\
0,002\end{array}$ & $\begin{array}{c}59,4 \pm 25,8 \\
61,7 \pm 22,8 \\
0,453\end{array}$ & $\begin{array}{c}64,7 \pm 15,7 \\
67,7 \pm 15,8 \\
0,145\end{array}$ \\
\hline
\end{tabular}

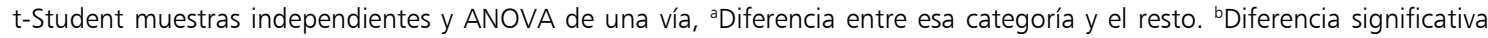
entre categorías. 


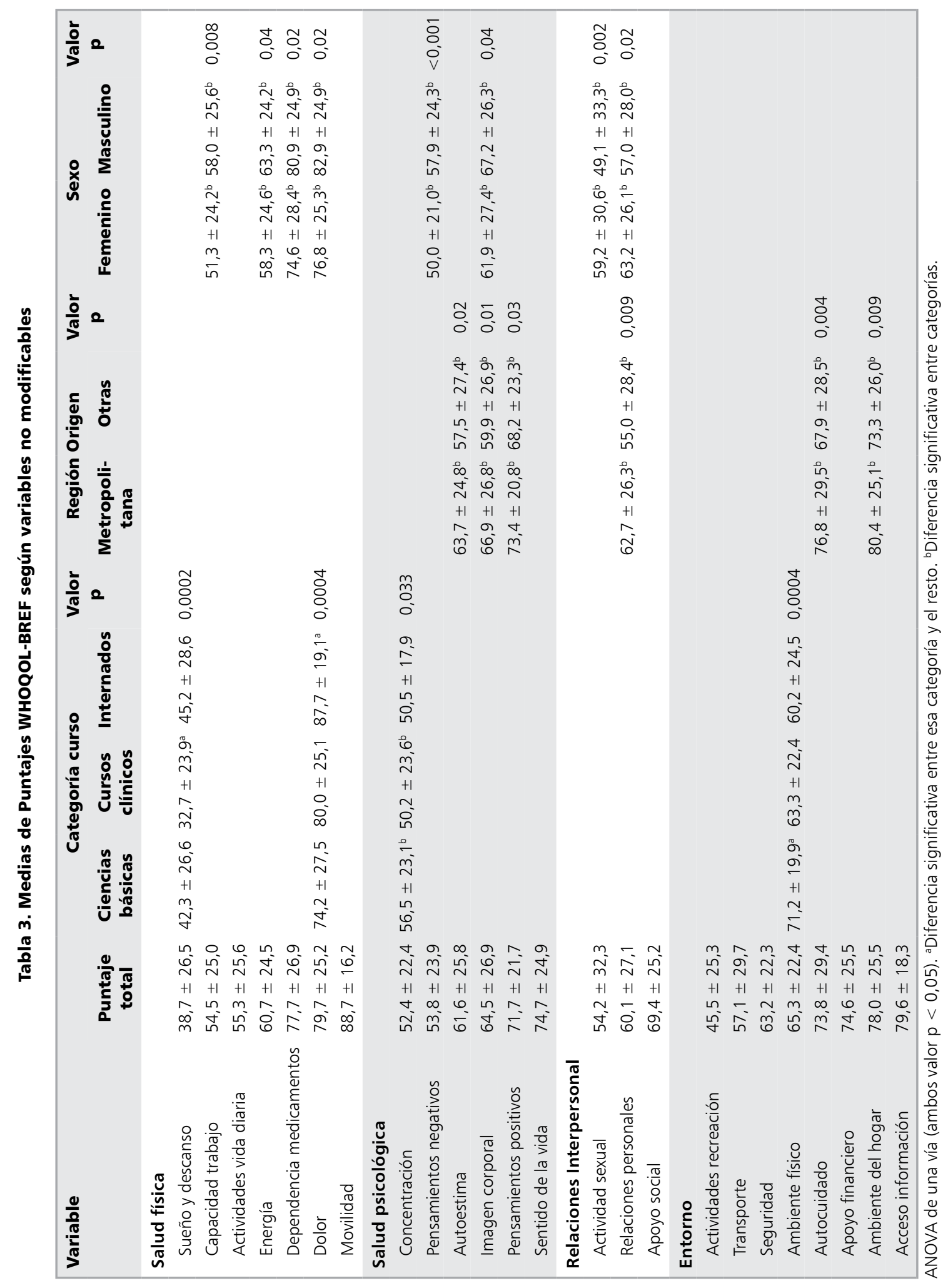


Tabla 4. Variables específicas de cada dominio de WHOQOL-BREF según variables modificables (Actividad Física y Consumo de Modafinilo)

\begin{tabular}{|c|c|c|c|c|c|c|}
\hline \multirow[t]{2}{*}{ Variable } & \multicolumn{4}{|c|}{ Actividad Física } & \multicolumn{2}{|c|}{ Consumo de modafinilo } \\
\hline & $\begin{array}{c}\text { Puntaje } \\
\text { total }\end{array}$ & Sí & No & $\mathbf{p}$ & No & $\mathbf{p}$ \\
\hline \multicolumn{7}{|l|}{ Salud física } \\
\hline Sueño y descanso & $38,7 \pm 26,5$ & $41,4 \pm 26,7$ & $34,1 \pm 25,5$ & 0,008 & & \\
\hline Capacidad trabajo & $54,5 \pm 25,0$ & $58,2 \pm 23,9$ & $48,1 \pm 25,9$ & $<0,001$ & $48,9 \pm 24,6 \quad 55,8 \pm 25,1$ & 0,038 \\
\hline Actividades vida diaria & $55,3 \pm 25,6$ & $59,2 \pm 24,9$ & $48,4 \pm 25,4$ & $<0,001$ & $48,9 \pm 27,1 \quad 56,7 \pm 25,1$ & 0,02 \\
\hline Energía & $60,7 \pm 24,5$ & $64,6 \pm 24,1$ & $53,9 \pm 23,7$ & $<0,001$ & & \\
\hline Dependencia medicamentos & $77,7 \pm 26,9$ & & & & $64,1 \pm 31,3 \quad 80,6 \pm 25,0$ & $<0,001$ \\
\hline Dolor & $79,7 \pm 25,2$ & & & & & \\
\hline Movilidad & $88,7 \pm 16,2$ & $89,8 \pm 16,9$ & $87,0 \pm 14,7$ & 0,045 & & \\
\hline \multicolumn{7}{|l|}{ Salud psicológica } \\
\hline Concentración & $52,4 \pm 22,4$ & $55,0 \pm 20,7$ & $47,9 \pm 24,7$ & 0,002 & $46,1 \pm 21,4 \quad 53,8 \pm 22,5$ & 0,009 \\
\hline Pensamientos negativos & $53,8 \pm 23,9$ & $57,3 \pm 21,7$ & $47,7 \pm 23,9$ & $<0,001$ & $46,4 \pm 23,4 \quad 55,4 \pm 22,6$ & 0,003 \\
\hline Autoestima & $61,6 \pm 25,8$ & $66,9 \pm 24,2$ & $52,4 \pm 26,1$ & $<0,001$ & & \\
\hline Imagen corporal & $64,5 \pm 26,9$ & $68,1 \pm 25,7$ & $58,2 \pm 28,0$ & $<0,001$ & $56,1 \pm 28,4 \quad 66,3 \pm 26,4$ & 0,004 \\
\hline Pensamientos positivos & $71,7 \pm 21,7$ & $75,2 \pm 19,5$ & $65,6 \pm 24,0$ & $<0,001$ & & \\
\hline Sentido de la vida & $74,7 \pm 24,9$ & $77,7 \pm 22,6$ & $69,6 \pm 28,0$ & 0,001 & & \\
\hline \multicolumn{7}{|l|}{ Relaciones interpersonales } \\
\hline Actividad sexual & $54,2 \pm 32,3$ & & & & & \\
\hline Relaciones personales & $60,1 \pm 27,1$ & $63,4 \pm 26,2$ & $54,5 \pm 28,1$ & 0,002 & & \\
\hline Apoyo social & $69,4 \pm 25,2$ & $73,0 \pm 22,9$ & $63,3 \pm 27,9$ & $<0,001$ & & \\
\hline \multicolumn{7}{|l|}{ Entorno } \\
\hline Actividades recreación & $45,5 \pm 25,3$ & $48,4 \pm 24,9$ & $40,6 \pm 25,3$ & 0,003 & & \\
\hline Transporte & $57,1 \pm 29,7$ & $60,0 \pm 28,2$ & $52,2 \pm 31,9$ & 0,012 & & \\
\hline Seguridad & $63,2 \pm 22,3$ & $66,44 \pm 21,2$ & $57,5 \pm 23,3$ & $<0,001$ & & \\
\hline Ambiente físico & $65,3 \pm 22,4$ & $69,0 \pm 21,0$ & $58,8 \pm 23,6$ & $<0,001$ & & \\
\hline Autocuidado & $73,8 \pm 29,4$ & & & & & \\
\hline Apoyo financiero & $74,6 \pm 25,5$ & & & & & \\
\hline Ambiente del hogar & $78,0 \pm 25,5$ & & & & & \\
\hline Acceso información & $79,6 \pm 18,3$ & $81,4 \pm 16,9$ & $76,4 \pm 20,5$ & 0,007 & & \\
\hline
\end{tabular}

t-Student muestras independientes.

4. Calidad de Vida según la variable "actividad física"

Los estudiantes que señalan practicar deporte, en comparación a los sedentarios, presentan una mayor puntuación en CV, satisfacción de salud y cada uno de los dominios evaluados (Tabla 3). $\mathrm{Al}$ analizar por ítem en cada dominio, quienes realizan deporte tienen mejores puntajes en todas las preguntas, siendo significativo en $69 \%$ de los ítems que abarcan salud física, psicológica, relaciones interpersonales y entorno (Tabla 4).

5. Calidad de Vida según la variable "consumo de modafinilo"

Los estudiantes que han consumido moda- finilo durante el último año presentan puntajes significativamente más bajos en la pregunta ¿cuán satisfecho está con su salud?, salud física y psicológica y salud física (Tabla 2). En salud psicológica, manifestaron menor capacidad de concentración, mayor frecuencia de pensamientos negativos y peor imagen corporal (Tabla 4).

6. Calidad de Vida según la variable "Estado Nutricional"

En las preguntas ¿cómo puntuaría su calidad de vida? y ¿cuán satisfecho está con su salud?, los alumnos con peso normal presentaron puntajes significativamente mayores que los alumnos con sobrepeso y obesidad $(\mathrm{p}<0,015)$. 
7. Calidad de Vida según la variable "Tabaquismo" $y$ Drogas

No se encontró diferencias significativas entre los alumnos según consumo de tabaco y drogas.

8. Análisis multivariado de calidad de vida

El análisis de regresión lineal multivariada para la pregunta ¿cómo puntuaría su calidad de vida?, mostró una relación directa con actividad fisica $(\mathrm{p}<0,001)$ e inversa con el IMC $(\mathrm{p}=0,004)$, cursos clínicos e internados $(\mathrm{p}<0,001$ y 0,003 respectivamente) (Tabla 5). Para la pregunta ¿qué tan satisfecho esta con su salud?, las variables actividad física, cursos clínicos, sobrepeso y obesidad, mostraron una asociación indirecta (Tabla 5).

En relación a salud física, ser mujer y consumir modafinilo se asoció inversamente a la salud físi$\mathrm{ca}$, mientras que la práctica de actividad física se asoció directamente con este dominio (Tabla 6). El consumo de modafinilo, sobrepeso y obesidad se asociaron inversamente a los puntajes de salud psicológica, mientras que la actividad física mostró una asociación directa (Tabla 6).

En relaciones interpersonales las mujeres y el

Tabla 5. Modelos de Regresión lineal múltiple para WHOQOL-BREF Estudiantes de Medicina BREF: Calidad de Vida y Satisfacción en Salud

\begin{tabular}{|c|c|c|c|c|}
\hline \multirow[t]{2}{*}{ Variables independientes } & \multicolumn{2}{|c|}{ Calidad de Vida $R^{2}$ 0,11 } & \multicolumn{2}{|c|}{ Satisfacción en Salud $R^{2} 0,12$} \\
\hline & $\beta$ (ES) & Valor $\mathbf{p}$ & $\beta$ (ES) & Valor $\mathbf{p}$ \\
\hline Sexo femenino & $-0,17$ & 0,949 & $-2,57$ & 0,291 \\
\hline Otra región origen & $-1,95$ & 0,396 & $-3,09$ & 0,224 \\
\hline Cursos clínicos & $-10,15$ & $<0,001$ & $-5,77$ & 0,043 \\
\hline Internados & $-9,10$ & 0,003 & $-1,31$ & 0,733 \\
\hline IMC bajo peso* & $-10,61$ & 0,173 & $-9,11$ & 0,29 \\
\hline IMC sobrepeso* & $-7,97$ & 0,004 & $-10,56$ & 0,001 \\
\hline IMC obesidad* & $-12,52$ & 0,056 & $-25,33$ & $<0,001$ \\
\hline Actividad física & 8,34 & $<0,001$ & 9,17 & $<0,001$ \\
\hline Modafinilo & $-0,79$ & 0,788 & $-6,11$ & 0,059 \\
\hline
\end{tabular}

WHOQOL-BREF: quality of life questionnaire proposed by the World Health Organization; IMC: Índice de masa corporal (m/ $\mathrm{kg}^{2}$ ). *Los valores de IMC se compararon con IMC normal.

Tabla 6. Modelos de Regresión lineal múltiple para dominios de WHOQOL-BREF Estudiantes de Medicina BREF

\begin{tabular}{|c|c|c|c|c|c|c|c|c|}
\hline \multirow[t]{2}{*}{$\begin{array}{l}\text { Variables } \\
\text { independientes }\end{array}$} & \multicolumn{2}{|c|}{$\begin{array}{c}\text { Salud física } \\
R^{2} 0,10\end{array}$} & \multicolumn{2}{|c|}{$\begin{array}{c}\text { Salud psicológica } \\
\mathbf{R}^{2} 0,11\end{array}$} & \multicolumn{2}{|c|}{$\begin{array}{l}\text { Relaciones interpersonales } \\
\qquad \mathbf{R}^{2} 0,04\end{array}$} & \multicolumn{2}{|c|}{$\begin{array}{l}\text { Entorno } \\
R^{2} 0,11\end{array}$} \\
\hline & $\beta$ (ES) & Valor $\mathbf{p}$ & $\beta$ (ES) & Valor $p$ & $\beta$ (ES) & Valor $p$ & $\beta$ (ES) & Valor $p$ \\
\hline Sexo femenino & $-4,97$ & 0,001 & $-2,56$ & 0,14 & 4,83 & 0,040 & $-0,19$ & 0,905 \\
\hline Otra región origen & $-1,63$ & 0,277 & $-3,55$ & 0,051 & $-4,42$ & 0,070 & $-3,52$ & 0,035 \\
\hline Cursos clínicos & $-2,29$ & 0,17 & $-2,06$ & 0,309 & $-2,80$ & 0,309 & $-2,38$ & 0,202 \\
\hline Internados & 2.94 & 0,131 & 0,2 & 0,934 & $-0,24$ & 0,941 & $-2,32$ & 0,289 \\
\hline IMC bajo peso* & $-3,27$ & 0,515 & $-0,5$ & 0,934 & $-0,76$ & 0,927 & $-3,67$ & 0,513 \\
\hline IMC sobrepeso* & $-2,47$ & 0,161 & $-5,24$ & 0,015 & $-2,29$ & 0,432 & $-3,63$ & 0,066 \\
\hline IMC obesidad* & $-7,41$ & 0,09 & $-17,45$ & 0,001 & $-8,31$ & 0,249 & $-4,51$ & 0,356 \\
\hline Actividad física & 5,98 & $<0,001$ & 7,92 & $<0,001$ & 6,80 & 0,005 & 4,79 & 0,004 \\
\hline Modafinilo & $-5,36$ & 0,005 & $-5,16$ & 0,025 & $-0,51$ & 0,870 & $-1,62$ & 0,445 \\
\hline
\end{tabular}

WHOQOL-BREF: quality of life questionnaire proposed by the World Health Organization; IMC: Índice de masa corporal (m/ $\mathrm{kg}^{2}$ ). *Los valores de IMC se compararon con IMC normal. 
consumo de modafinilo presentaron una relación inversa $(\mathrm{p}<0,005)$ y actividad física una relación directa $(\mathrm{p}<0,001)$. Por último, el entorno, al igual que las otras dimensiones, presenta asociación positiva con actividad física $(\mathrm{p}=0,004)$ y asociación negativa con provenir de una región geográfica distinta a la RM ( $\mathrm{p}=0,035)$ (Tabla 6).

\section{Análisis factorial}

El alpha de Cronbach's fue mayor a 0,7 en todas las dimensiones, valor que es considerado como aceptable.

\section{Discusión}

El principal hallazgo de este estudio fue que la CV de los estudiantes de Medicina de una escuela universitaria chilena se modifica según variables intrínsecas y extrínsecas a los sujetos de estudio. El mayor efecto negativo se observó en la transición de cursos de Ciencias Básicas a Cursos Clínicos, en alumnos con origen fuera de la RM, en alumnos que presentan consumo de modafinilo y en alumnos con sobrepeso. Los estudiantes que realizan actividad física obtuvieron mejores puntajes de CV.

Países como China y Brasil han realizado investigaciones similares, utilizando el cuestionario WHOQOL-BREF para medir CV en estudiantes de Medicina ${ }^{1,16}$. En comparación al estudio realizado en China, los resultados de nuestro estudio muestran globalmente mejores puntajes de CV. Las únicas preguntas que mostraron menores puntajes estuvieron relacionadas con las actividades de la vida diaria ( 55,3 vs 58 ) y los pensamientos negativos ( 53,8 vs 58 ). Respecto al estudio brasilero, los puntajes de los Salud física y psicológica fueron similares a los obtenidos en este estudio, mientras que en relaciones interpersonales los brasileros obtuvieron mayores puntajes $(68,9$, vs 61,3$)$. En entorno físico los puntajes globales de este estudio fueron mayores (67,2 vs 58).

$\mathrm{Al}$ analizar los puntajes en cada dominio del cuestionario, según los factores que pudiesen influir en la $\mathrm{CV}$, se encontró una asociación negativa en los puntajes obtenidos en el $C V$ global, satisfacción de salud y salud física, donde los Cursos Clínicos presentaron menores puntajes respecto a las preguntas que consideran el sueño y la concentración. Estos resultados son concordantes con un estudio realizado en Brasil, donde los autores concluyen que los síntomas depresivos fueron más prevalentes en el período medio de la carrera, asociado al período de transición entre los cursos de Ciencias Básicas y los Cursos Clínicos, siendo éstos últimos asociados a mayor práctica clínica, en la cual los estudiantes se ven expuestos a pacientes, enfermedad y muerte ${ }^{2,17,18}$. A su vez, en los Cursos Clínicos se solicita por parte de los docentes, una mayor integración del conocimiento requiriendo mayor estudio, dedicación y esfuerzo, lo cual puede aumentar el estrés psicológico ${ }^{19}$. Estudios previos han demostrado una asociación entre la transición a Cursos Clínicos y una menor estabilidad emocional, la cual se manifiesta en ansiedad e inseguridad ${ }^{20-22}$. A pesar de lo anterior, existe evidencia contraria, que sostiene una mayor prevalencia de estados de peor salud mental en alumnos de primer año $(37 \%)$, decreciendo en cuarto y quinto año $(31 \%$ y $22 \%$, respectivamente $)^{23}$.

En cuanto a la dimensión entorno, los cursos correspondientes al Internado obtuvieron puntajes significativamente menores. Esto podría estar relacionado a las condiciones ambientales de trabajo, como el espacio físico para trabajar y descansar, el lugar para comer y condiciones de la residencia de los turnos nocturnos.

El factor región geográfica de origen influyó en los puntajes de CV. Existe una relación negativa en la salud psicológica, relaciones interpersonales y entorno de aquellos alumnos provenientes de otras regiones del país. Esto se podría explicar dado que el origen geográfico fuera de la RM conlleva a una adaptación en un entorno diferente, incluyendo la integración en el ámbito social y cultural. Al realizar un análisis de los puntajes obtenidos en las preguntas específicas del cuestionario, este grupo de estudiantes obtuvo menores puntajes en autoestima, imagen corporal y capacidad de disfrutar la vida, por lo que requiere una mayor atención para eventuales intervenciones en salud y prevención de patología mental.

Respecto al sexo, las mujeres obtuvieron peores puntajes en el dominio de salud física, específicamente en las áreas de capacidad de trabajo, energía, dependencia de medicamentos y dolor, por otra parte, en el análisis de las preguntas específicas del dominio salud psicológica, las mujeres presentaron mayor prevalencia de pensamientos negativos y peor imagen corporal. Estos resultados son concordantes a los resultados obtenidos en el 
Calidad de vida de estudiantes de Medicina y factores asociados - L. Irribarra T. et al

estudio de Chazan et $\mathrm{al}^{16}$, donde la variable que influía más negativamente en el dominio salud física fue el sexo femenino, quienes a su vez también tenían peores puntajes en el dominio salud psicológica. Sin embargo, las mujeres presentaron mejores puntajes en las relaciones interpersonales, resultado concordante al estudio de Zhang et $\mathrm{al}^{1}$, esto podría estar explicado por la mayor habilidad de las mujeres en la construcción de relaciones interpersonales ${ }^{24}$.

Es importante destacar la relevancia de tres variables modificables evaluadas: actividad física, sobrepeso/obesidad y uso de modafinilo. El sedentarismo afectó negativamente a todos los dominios evaluados, siendo concordante con estudios similares que señalan que la actividad física y peso corporal son importantes predictores de salud mental y física ${ }^{5,25,26}$. Esto refuerza la importancia de la actividad física y control del peso corporal como factores protectores de la CV, estando ambas variables relacionadas entre ellas ${ }^{26}$. Intervenciones que promueva la actividad física y evitar el sobrepeso y obesidad podrían afectar positivamente el bienestar y desempeño académico ${ }^{27,28}$.

El modafinilo, por su parte, afecta negativamente los dominios de salud física y psicológica, lo cual podría estar relacionado principalmente con las causas que llevan a consumirlo, como deprivación de sueño y fatiga ${ }^{29}$.

Este estudio describe la CV en estudiantes de Medicina en una universidad chilena, entregando bases para futuras intervenciones positivas a nivel local. Los focos de éstas debiesen considerar incentivar la práctica deportiva, además de poner especial atención en los estudiantes de los Cursos Clínicos y aquellos con un origen geográfico diferente a la RM. Estudios previos han demostrado que la exposición temprana de los estudiantes al entrenamiento clínico, la metodología de aprendizaje basado en problemas, y la utilización de herramientas para manejar el estrés, les permitiría disminuir la fatiga y ansiedad asociada a los Cursos Clínicos $^{12,30}$.

Este estudio presenta algunas limitaciones, ya que al haber sido aplicada una encuesta de forma online, el porcentaje de respuesta fue menor a la población total de los estudiantes. De la misma forma, la etapa de formación Internados obtuvo menores tasas de respuesta en comparación a las otras etapas, probablemente asociado a la carga docente-asistencial de este período. El diseño del estudio sólo permite asociar las variables de interés, por lo que no es posible establecer causalidad en quienes refirieron no realizar actividad física, con sobrepeso u obesidad, cuyos puntajes en el cuestionario de $\mathrm{CV}$ fueron significativamente menores.

\section{Conclusiones}

La CV de los estudiantes de Medicina de una universidad chilena varía según las etapas de formación, región geográfica de origen de los estudiantes, sexo, actividad física, sobrepeso y consumo de modafinilo. El efecto negativo más significativo en las etapas de formación se observó en la transición de cursos de Ciencias Básicas a Cursos Clínicos, el origen geográfico fuera de la Región Metropolitana, el sobrepeso y obesidad, y el uso de modafinilo, mientras que el efecto positivo más relevante se observó asociado a la práctica de actividad física.

Agradecimientos: Agradecemos al Dr. Gerardo Robles por su colaboración en la revisión bibliográfica y recolección de datos para este estudio.

\section{Referencias}

1. Zhang Y, Qu B, Lun S, Wang D, Guo Y, Liu J. Quality of life of medical students in China: a study using the WHOQOL-BREF. PloS One 2012; 7 (11): e49714.

2. Dyrbye LN, Thomas MR, Eacker A, Harper W, Massie FS, Power DV, et al. Race, ethnicity, and medical student well-being in the United States. Arch Intern Med 2007; 167 (19): 2103-9.

3. Samaranayake CB, Fernando AT. Satisfaction with life and depression among medical students in Auckland, New Zealand. N Z Med J 2011; 124 (1341): 12-7.

4. Henning MA, Krägeloh CU, Hawken SJ, Zhao Y, Doherty I. The quality of life of medical students studying in New Zealand: a comparison with nonmedical students and a general population reference group. Teach Learn Med 2012; 24 (4): 334-40.

5. Lins L, Carvalho FM, Menezes MS, Porto-Silva L, Damasceno H. Health-related quality of life of students from a private medical school in Brazil. Int J Med Educ 2015; 6: 149-54.

6. The World Health Organization Quality of Life assessment (WHOQOL): position paper from the World 
Health Organization. Soc Sci Med 1982. 1995; 41 (10): 1403-9.

7. Hojat M, Robeson M, Damjanov I, Veloski JJ, Glaser K, Gonnella JS. Students' psychosocial characteristics as predictors of academic performance in medical school. Acad Med J Assoc Am Med Coll 1993; 68 (8): 635-7.

8. American Board of Internal Medicine. American Board of Internal Medicine. Project Professionalism. [Internet]. 1994. Disponible en:_http://www.abim.org/ resources/publications/professionalism.pdf

9. Benítez GC, Quintero BJ, Torres BR. Prevalencia de riesgo de trastornos psiquiátricos en estudiantes de pregrado de la Escuela de Medicina de la P. Universidad Católica de Chile. Rev Med Chile 2001; 129 (2): 173-8.

10. Santander TJ, Romero SMI, Hitschfeld AMJ, Zamora AV. Prevalencia de ansiedad y depresión entre los estudiantes de medicina de la Pontificia Universidad Católica de Chile. Rev Chil Neuro-Psiquiat 2011; 49 (1): 47-55.

11. Marty M, Lavín GM, Figueroa MM, Larraín de la C D, Cruz MC. Prevalencia de estrés en estudiantes del área de la salud de la Universidad de los Andes y su relación con enfermedades infecciosas. Rev Chil Neuro-Psiquiat 2005; 43 (1): 25-32.

12. Síndrome de burnout y factores asociados en estudiantes de una escuela de medicina de Chile. Patricio Alfaro-Toloza, Romina Olmos-de-Aguilera, Maximiliano Fuentealba, Egidio Céspedes-González. Ciencia e Investigación Medico Estudiantil Latinoamericana 18 (2).

13. Skevington SM, Lotfy M, O'Connell KA. The World Health Organization's WHOQOL-BREF quality of life assessment: Psychometric properties and results of the international field trial. A Report from the WHOQOL Group. Qual Life Res 2004; 13: 299-310.

14. Bentler PM. Comparative fit indexes in structural models. Psychol Bull 1990; 107: 238-46.

15. Steiger J. Structural model evaluation and modification: an interval estimation approach. Multivariate Behav Res 1990; 25: 173-80.

16. Chazan ACS, Campos MR, Portugal FB. Quality of life of medical students at the State University of Rio de Janeiro (UERJ), measured using Whoqol-bref: a multivariate analysis. Cienc Saude Coletiva 2015; 20 (2): 547-56.

17. Paro HBMS, Morales NMO, Silva CHM, Rezende CHA, Pinto RMC, Morales RR, et al. Health-related quality of life of medical students. Med Educ 2010; 44 (3): 227-35.

18. Houpy JC, Lee WW, Woodruff JN, Pincavage AT. Medical student resilience and stressful clinical events during clinical training. Med Educ Online [Internet]. 2017; 22
(1). Disponible en: https://www.ncbi.nlm.nih.gov/pmc/ articles/PMC5419301/

19. Dyrbye LN, Thomas MR, Shanafelt TD. Medical Student Distress: Causes, Consequences, and Proposed Solutions. Mayo Clin Proc 2005; 80 (12):1613-22.

20. Compton MT, Carrera J, Frank E. Stress and depressive symptoms/dysphoria among US medical students: results from a large, nationally representative survey. J Nerv Ment Dis 2008; 196 (12): 891-7.

21. Pitkälä KH, Mäntyranta T. Feelings related to first patient experiences in medical school. A qualitative study on students' personal portfolios. Patient Educ Couns 2004; 54 (2): 171-7.

22. Dahlin M, Joneborg N, Runeson B. Stress and depression among medical students: a cross-sectional study. Med Educ 2005; 39 (6): 594-604.

23. Guthrie EA, Black D, Shaw CM, Hamilton J, Creed FH, Tomenson B. Embarking upon a medical career: psychological morbidity in first year medical students. Med Educ 1995; 29 (5): 337-41.

24. Palchykov V, Kaski K, Kertész J, Barabási A-L, Dunbar RIM. Sex differences in intimate relationships. Sci Rep [Internet]. 2012;2. Disponible en: https://www.ncbi.nlm. nih.gov/pmc/articles/PMC3329793/

25. Jamali A, Tofangchiha S, Jamali R, Nedjat S, Jan D, Narimani A, et al. Medical students' health-related quality of life: roles of social and behavioural factors. Med Educ 2013; 47 (10): 1001-12.

26. Stephens SK, Cobiac LJ and Veerman JL. Improving diet and physical activity to reduce population prevalence of overweight and obesity: an overview of current evidence. Prev Med 2014, p. 167-78.

27. Al-Drees A, Abdulghani $H$, Irshad $M$, Baqays AA, Al-Zhrani AA, Alshammari SA, et al. Physical activity and academic achievement among the medical students: A cross-sectional study. Med Teach 2016; 38 Suppl 1: S66-72.

28. Morris A, Do D, Gottlieb-Smith R, Ng J, Jain A, Wright $S$, et al. Impact of a fitness intervention on medical students. South Med J 2012; 105 (12): 630-4.

29. Cortese S, Adamo N, Mohr-Jensen C, Hayes AJ, Bhatti $S$, Carucci S, et al. Comparative efficacy and tolerability of pharmacological interventions for attention-deficit/ hyperactivity disorder in children, adolescents and adults: protocol for a systematic review and network meta-analysis. BMJ Open 2017; 7 (1): e013967.

30. Moffat KJ, McConnachie A, Ross S, Morrison JM. First year medical student stress and coping in a problem-based learning medical curriculum. Med Educ 2004; 38 (5): 482-91. 